NOTICE: this is the author's version of a work that was accepted for publication in Nonlinear Analysis: Hybrid Systems. Changes resulting from the publishing process, such as peer review, editing, corrections, structural formatting, and other quality control mechanisms may not be reflected in this document. Changes may have been made to this work since it was submitted for publication. A definitive version was subsequently published in Nonlinear Analysis: Hybrid Systems, Vol. 14 (2014). DOI: 10.1016/j.nahs.2014.05.004 


\title{
Global Stabilization of Switched Control Systems with Time Delay
}

\author{
Yi Zhang ${ }^{1}$, Min Wang ${ }^{1}$, Honglei $\mathrm{Xu}^{2}$ and Kok Lay Teo ${ }^{2}$ \\ ${ }^{1}$ College of Science, Department of Mathematics, \\ China University of petroleum-Beijing, China, 102249. \\ ${ }^{2}$ Department of Mathematics and Statistics, \\ Curtin University, Perth, WA 6845,Australia
}

\begin{abstract}
In this paper, the stabilization problem of switched control system$\mathrm{s}$ with time delay is investigated for both linear and nonlinear cases. First, a new global stabilizability concept with respect to state feedback and switching law is given. Then, based on multiple Lyapunov functions and delay inequalities, the state feedback controller and the switching law are devised to make sure that the resulting closed-loop switched control systems with time delay are globally asymptotically stable and exponentially stable.
\end{abstract}

Key words: Switched control systems; stabilizability; time delay.

\section{Introduction}

A switched system is a hybrid system comprised of continuous-time or discrete-time subsystems and a rule that supervises the switching between subsystems. Switched systems can be found in many areas, such as comput-er science, control systems, electrical engineering and technology, automotive industry, and air traffic management and control [1]-[4]. For switched system$\mathrm{s}$, most important and challenging problems are on stability and stabilization (i.e., is it possible to design (or find) a switching law under which the resulting switched systems are stable?). Hence, the recent focus of switched control 
systems is on the design of a switched law and a controller under which the controlled systems are stable. In the last two decades, stability analysis of switched systems and switching control design have attracted considerable attention among control theorists, computer scientists, mathematicians and practicing engineers. Many interesting and important results have been established. See, for example, [3]-[14] and the references therein.

On the other hand, time delay phenomenon exists in many practical switched systems, see, for example, [9]-[10], [14]-[15]. Stability and stabilization problem is also an important and challenging problem for switched systems with time delay. In [15], exponential stability of switched system$\mathrm{s}$ with time-delay is established based on average dwell time and Lyapunov functions methods. Using a multiple Lyapunov function method, exponential stability of some special linear switched system is investigated in [16]. Moreover, a linear matrix inequality (LMI) method is applied to study the stability problem of switched systems in [17]-[20]. In [21], asymptotic stability and stabilization of a class of switched control systems is studied, where a delaydependent stability criterion is formulated in term of LMIs by using quadratic Lyapunov functions and inequality analysis technique. For discrete-time systems, some interesting results can be found in [4], [22] and [23]. Other methods, such as dwell time and average dwell time, are used in the study of switched systems. For example, stability of some slow-switched control systems has been studied in [24], and stabilization problems for switched systems have been discussed in [25]-[28], [38], [39].

For the results mentioned above, the switching signal does not involve time delay. However, in real world, a switched control system may have several controllers and not all the controllers are required to switch at the same time. This class of systems can be described by switched control systems in which the switching signal has time delay. These switched control systems are much more complex than a conventional switched system and hence only few works are available in the literature, such as, [29] and [30], where some sufficient conditions for stability are derived for some switched linear systems with time delay appearing in the switching signal. It appears that no results on the stabilization problem are available in the literature for nonlinear switched control systems with distributed time delays and time delay appearing in the switching signal. This has motivated our research. In addition, our results obtained can be applied to the cases with asynchronous switching in actual operation, such as the stabilization of chemical systems [36] and multi-agent systems [33]-[35]. The asynchronous switching in sys- 
tems is always caused by the controller lags of the system.

In this paper, we consider static state feedback control for nonlinear switched system with distributed time delays and with time delay appear-ing in the switching signal. In this study, the time delay of the state may be different from the time delay appearing in the switching signal of the feedback controller. We first introduce some new concepts on stabilization in relation to controller and switching law. Then, by using the method of Lyapunov functions and delay inequalities, some delay-dependent conditions are derived for the design of state feedback controller and a switching law to guarantee the stability of the resulting closed-loop switched control sys-tems. The advantages of this paper are twofold. First, the system model includes both integral terms, called distributed delay, and asynchronous con-trol time lags. This model can cover most of the existing models for switched linear/ nonlinear systems. Second, the stability issue under investigation is with respect to both state feedback control and switching signal, which is a much more general problem than previous results.

The outline of the paper is as follows. Section 2 presents some definitions and some technical lemmas needed for the proof of the main results. The design of a switching law for global asymptotical or exponential stabilizability of linear and nonlinear switched control systems are obtained in Section 3 and Section 4, respectively. Finally, some concluding remarks are made in Section 5.

\section{Preliminaries}

The following switched control system is considered:

$$
\dot{x}(t)=A_{\sigma(t)} x(t)+B_{\sigma(t)} u+f_{\sigma(t)}\left(x(t), x_{t}\right),
$$

where $x(t) \in R^{n}, u \in R^{n}$ is state feedback control, $A_{\sigma(t)} \in R^{n \times n}$, and $B_{\sigma(t)} \in R^{n \times n}$ are state matrices, the right continuous function $\sigma(t): R \rightarrow$ $\Theta=\{1,2, \cdots, N\}$ is the switching signal, $x_{t} \in C_{\tau}=\left\{\varphi \mid \varphi \in C\left([-\tau, 0], R^{n}\right)\right\}$, $\tau>0$ is a constant and $f_{\sigma(t)} \in C\left(R^{n} \times C_{\tau}, R^{n}\right)$ satisfy $f_{\sigma(t)}(0,0)=0$.

In this paper, $N_{+}=\{1,2, \cdots\}, R_{+}=[0,+\infty), E$ is an identity matrix of appropriate dimension, $|A|$ denotes the usual norm of a matrix $A \in R^{n \times m}$, $\left\|x_{t}\right\|=\sup _{t-\tau \leq \theta \leq t}|x(\theta)|$ denotes the sup norm of the function $x_{t} \in C_{\tau}$. If $A \in R^{n \times n}, \lambda(\bar{A})$ denotes eigenvalue of $A$. Let $\sigma=\left\{\left(i_{1}, t_{1}\right), \cdots,\left(i_{k}, t_{k}\right), \cdots\right\}$ 
be the switching law, meaning that when $t \in\left[t_{k}, t_{k+1}\right)$, the $i_{k+1}^{t h}$ subsystem is active, i.e.

$$
\dot{x}(t)=A_{k+1} x(t)+B_{k+1} u+f_{k+1}\left(x(t), x_{t}\right), \quad t \in\left[t_{k}, t_{k+1}\right) .
$$

Let $x\left(t, t_{0}, \varphi, \sigma\right)$ be the solution of system (1) under the switching law $\sigma$, starting from $\left(t_{0}, \varphi\right)$.

It is well known that, a switching law often plays an important role in the study of the stability of a switched system. Even if the subsystems are stable, different switching laws may results in totally different properties of the overall switched systems. In this paper, our focus is on to design a controller $u$ and a switching law $\sigma$ so that the resulting closed-loop switched systems are guaranteed to possess desired properties.

Next, we propose some concepts on the stabilization for the switched control system.

Definition 2.1. Consider the switched control system (1).

1) It is said to be stabilizable with respect to (w.r.t) the state feedback control $u$ and the switching law $\sigma$ (SWUS) if it is stable under the state feedback $u$ and the switching law $\sigma$. That is, for any $\varepsilon>0$, there exists a $\delta>0$, such that for any $\varphi \in C_{\tau},\|\varphi\|<\delta$ implies $\left\|x\left(t, t_{0}, \varphi, \sigma\right)\right\|<\varepsilon$.

2) It is said to be asymptotical stabilizable under the feedback control u and the switching law $\sigma$ (ASWUS) if it is SWUS and there exists a $\delta>0$ such that for any $\varphi \in C_{\tau},\|\varphi\|<\delta$ implies

$$
\lim _{t \rightarrow+\infty} x\left(t, t_{0}, \varphi, \sigma\right)=0 .
$$

3) It is said to be globally asymptotical stabilizable under the feedback control $u$ and the switching law $\sigma$ (GASWUS) if it is ASWUS and for any $\varphi \in C_{\tau}$, has

$$
\lim _{t \rightarrow+\infty} x\left(t, t_{0}, \varphi, \sigma\right)=0 .
$$

4) It is said to be exponential stabilizable under the feedback control $u$ and the switching law $\sigma$ (ESWUS) if there exist constants $M>0, \delta>$ $0, \lambda>0$, such that for any $\varphi \in C_{\tau},\|\varphi\|<\delta$ implies

$$
\left|x\left(t, t_{0}, \varphi, \sigma\right)\right| \leq M e^{-\lambda\left(t-t_{0}\right)}, \quad t \geq t_{0} .
$$


5) It is said to be globally exponential stabilizable under the feedback control $u$ and the switching law $\sigma$ (GESWUS) if there exist constants $M>$ $0, \lambda>0$, such that for any $\varphi \in C_{\tau}$, it holds that

$$
\left|x\left(t, t_{0}, \varphi, \sigma\right)\right| \leq M\|\varphi\| e^{-\lambda\left(t-t_{0}\right)}, \quad t \geq t_{0} .
$$

Without loss of generality, we choose, in this paper, $t_{0}=0$ and denote $x(t, 0, \varphi, \sigma):=x(t)$.

To study the stabilizability of system (1), we need the following lemmas.

Lemma 2.1. (Gronwall inequality) Suppose that $g, u \in C\left(\left[t_{0}, t_{1}\right), R_{+}\right)$, and that $c$ is a nonnegative real constant. If

$$
u(t) \leq c+\int_{t_{0}}^{t} g(s) u(s) d s, \quad t \in\left[t_{0}, t_{1}\right]
$$

then

$$
u(t) \leq c e^{\int_{t_{0}}^{t} g(s) d s} .
$$

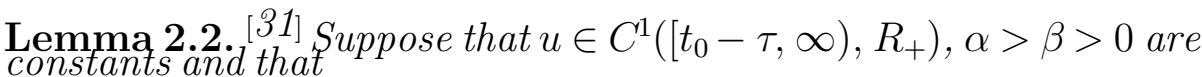

$$
u^{\cdot}(t) \leq-\alpha u(t)+\beta\left\|u_{t}\right\|, t \geq t_{0}
$$

Then, there exists a $\lambda^{\wedge}>0$ such that

$$
u(t) \leq\left\|u_{t_{0}}\right\| e^{-\hat{\lambda} \lambda\left(t-t_{0}\right)},
$$

where $\lambda^{\wedge}$ is the unique positive solution of the equation $\lambda^{\wedge}=\alpha-\beta e^{\lambda \tau}$.

Lemma 2.3. Suppose that $u \in C^{1}\left(\left[t_{0}-\tau, \infty\right), R_{+}\right), 0<\alpha \leq \beta$ are constants and that

$$
\dot{u}(t) \leq-\alpha u(t)+\beta\left\|u_{t}\right\|, \quad t \geq t_{0} .
$$

Then

$$
u(t) \leq\left\|u_{t_{0}}\right\| e^{\left(\beta e^{\alpha \tau}-\alpha\right)\left(t-t_{0}\right)} .
$$

Proof. From Lemma 2.2, we have

$$
\frac{d}{d t} e^{\alpha\left(t-t_{0}\right)} u(t) \leq e^{\alpha\left(t-t_{0}\right)} \beta\left\|u_{t}\right\|
$$


Integrating both sides gives

$$
\begin{aligned}
e^{\alpha\left(t-t_{0}\right)} u(t) & \leq u\left(t_{0}\right)+\beta \int_{t_{0}}^{t} e^{\alpha\left(s-t_{0}\right)}\left\|u_{s}\right\| d s \\
& \leq u\left(t_{0}\right)+\beta \int_{t_{0}}^{t} e^{\alpha \tau} \sup _{s-\tau \leq \theta \leq s}\left[e^{\alpha\left(\theta-t_{0}\right)} u(\theta)\right] d s .
\end{aligned}
$$

Since the right hand side of the inequality above is an increasing function, we have

$$
\sup _{t-\tau \leq \theta \leq t}\left[e^{\alpha\left(\theta-t_{0}\right)} u(\theta)\right] \leq\left\|u_{t_{0}}\right\|+\beta \int_{t_{0}}^{t} e^{\alpha \tau} \sup _{s-\tau \leq \theta \leq s}\left[e^{\alpha\left(\theta-t_{0}\right)} u(\theta)\right] d s .
$$

By Gronwall inequality, it follows that

$$
\sup _{t-\tau \leq \theta \leq t}\left[e^{\alpha\left(\theta-t_{0}\right)} u(\theta)\right] \leq\left\|u_{t_{0}}\right\| e^{\beta e^{\alpha \tau}\left(t-t_{0}\right)} .
$$

Since

$$
\sup _{t-\tau \leq \theta \leq t}\left[e^{\alpha\left(\theta-t_{0}\right)} u(\theta)\right] \geq e^{\alpha\left(t-t_{0}\right)} u(t)
$$

we have

$$
u(t) \leq\left\|u_{t_{0}}\right\| e^{\left(\beta e^{\alpha \tau}-\alpha\right)\left(t-t_{0}\right)} .
$$

Lemma 2.4. [32] Suppose that $Z \in R^{n \times n}$ is a positive definite matrix, $\epsilon>0$ is a scalar and $x, y \in R^{n}$. Then

$$
2 x^{T} y \leq x^{T} Z^{-1} x+y^{T} Z y,
$$

and, as a special case,

$$
2 x^{T} y \leq \epsilon^{-1} x^{T} x+\epsilon y^{T} y .
$$

\section{Linear Switched Control Systems}

In this section, we consider a linear switched control system, where the linear state feedback control is given by $u=K_{\gamma(t)} x$ and $f_{\sigma(t)}\left(x(t), x_{t}\right)=$ 
$C_{\sigma(t)} \int_{t-\tau}^{t} x(s) d s$, with $\gamma(t)=\sigma(t-\tau)$ and $C_{\sigma(t)} \in R^{n \times n}$. Then, the resulting switched system (1) becomes:

$$
\left\{\begin{array}{l}
\dot{x}(t)=A_{\sigma(t)} x(t)+B_{\sigma(t)} u+C_{\sigma(t)} \int_{t-\tau}^{t} x(s) d s, \\
u(t)=K_{\gamma(t)} x(t), \\
\gamma(t)=\sigma(t-\tau),
\end{array}\right.
$$

The closed-loop system may be written more specifically as:

$$
\dot{x}(t)=A_{\sigma(t)} x(t)+B_{\sigma(t)} K_{\sigma(t-\tau)} x(t)+C_{\sigma(t)} \int_{t-\tau}^{t} x(s) d s .
$$

System (2) in form of intero-differential equations contains not only integral terms but also delayed switching controllers. These controllers' description is consistent with that for the asynchronous switching controller in [36]. In addition, it can be seen that system (2) can cover the switching linear systems with asynchronous switching described in [36]. Our aim is to choose a control matrix $K_{\sigma(t-\tau)}$ and a switching law $\sigma$ such that the resulting switched system (2) is GASWUS or GESWUS.

To continue, we assume that the following conditions are satisfied.

\section{Assumption $\mathbb{A}_{1}$}

i) There exist $\alpha>0, \beta>0$, and $M \geq 1$ such that $\left\|e^{\left(A_{i}+B_{i} K_{i}\right) t}\right\| \leq M e^{-\alpha t}$, and ${ }_{\|} e^{\left(A_{i}+B_{i} K_{j}\right) t} \| \leq M e^{-\beta t}$ hold for all $i \neq j, i, j \in \Theta$;

ii) $\exists C>0$ such that $\left|C_{i}\right| \leq C$ for all $i \in \Theta$, and $-\lambda \triangleq C M \tau e^{\alpha \tau}-\alpha<0$;

iii) For the switching law $\sigma=\left\{\left(i_{1}, t_{1}\right), \cdots,\left(i_{m}, t_{m}\right), \cdots\right\}$, it holds that $t_{m}-t_{m-1} \geq 2 \tau, m \in N_{+}$.

Now, we have the following result.

Theorem 3.1. Suppose that Assumption $\mathbb{A}_{1}$ holds. Let $M_{m}:=m(2 \ln M+$ $\left.\tau\left(C M e^{\beta \tau}+\alpha+\lambda\right)\right), m \in N_{+}$. Then, the switched control system (2) is:

(a) SWUS, if there exists a constant $\tilde{M}$ such that the switching law $\sigma$ satisfies $M_{m}-\lambda t_{m} \leq \tilde{M}, m \in N_{+}$;

(b) GASWUS, if $\lim _{m \rightarrow \infty}\left(M_{m}-\lambda t_{m}\right)=-\infty$;

(c) GESWUS, if $\lim \sup _{m \rightarrow \infty} \frac{M_{m}-\lambda t_{m}}{t_{m}}<0$. 
Proof. Consider the closed-loop switched control system (2). For a given switching signal $\sigma=\left\{\left(i_{1}, t_{1}\right), \cdots,\left(i_{m}, t_{m}\right), \cdots\right\}$, when $t \in\left[0, t_{1}\right)$, the $i_{1}^{\text {th }}$ subsystem is active, meaning that

$$
x^{\cdot}(t)=A_{i_{1}} x(t)+B_{i_{1}} K_{i_{1}} x(t)+C_{i_{1}} \int_{t-\tau}^{t} x(s) d s .
$$

Its solution is

$$
x(t)=e^{\left(A_{i_{1}}+B_{i_{1}} K_{i_{1}}\right) t} x(0)+\int_{0}^{t} e^{\left(A_{i_{1}}+B_{i_{1}} K_{i_{1}}\right)(t-s)} \int_{s-\tau}^{s} C_{i_{1}} x(\psi) d \psi d s .
$$

Taking the norm on both sides, we

obtain

$$
|x(t)| \leq M e^{-\alpha t}|x(0)|+C M \tau \int_{0}^{t} e^{-\alpha(t-s)}\left\|x_{s}\right\| d s .
$$

Multiplying both sides by $e^{\alpha t}$, we have

$$
e^{\alpha t}|x(t)| \leq M|x(0)|+C M \tau \quad \int_{0}^{t} e^{\alpha s}\left\|x_{s}\right\| d s
$$

Since the right hand side of the inequality above is an increasing function, it follows that

$$
\begin{aligned}
e^{\alpha t}\left\|x_{t}\right\| \leq & e^{\alpha \tau} \sup _{t-\tau \leq \theta \leq t} \quad e^{\alpha \theta}|x(\theta)| \\
& \leq M e^{\alpha \tau}\|\varphi\|+e^{\alpha \tau} C M \tau \quad \int_{0}^{t} e^{\alpha s}\left\|x_{s}\right\| d s .
\end{aligned}
$$

Thus, by Lemma 2.1, we obtain

$$
\left\|x_{t}\right\| \leq M e^{\alpha \tau}\|\varphi\| e^{-\lambda t}, t \in\left[0, t_{1}\right) .
$$

From this inequality, it follows from the continuity property of the solution that

$$
\left\|x_{t_{1}}\right\| \leq M e^{\alpha \tau}\|\varphi\| e^{-\lambda t_{1}} .
$$

Now, for the case $t \in\left[t_{m-1}, t_{m-1}+\tau\right)$, where $m \in N_{+}$, and $m>1$. The active subsystem is:

$$
x \cdot(t)=A_{i_{m}} x(t)+B_{i_{m}} K_{i_{m-1}} x(t)+C_{i_{m}} \int_{t-\tau}^{t} x(s) d s
$$


and for $t \in\left[t_{m-1}+\tau, t_{m}\right)$, the active subsystem is:

$$
\dot{x}(t)=A_{i_{m}} x(t)+B_{i_{m}} K_{i_{m}} x(t)+C_{i_{m}} \int_{t-\tau}^{t} x(s) d s .
$$

By a similar argument, we can show that

$$
\left\|x_{t}\right\| \leq \begin{cases}M e^{\beta \tau}\left\|x_{t_{m-1}}\right\| e^{\left(C M \tau e^{\beta \tau}-\beta\right)\left(t-t_{m-1}\right)}, & t \in\left[t_{m-1}, t_{m-1}+\tau\right), \\ M e^{\alpha \tau}\left\|x_{t_{m-1}+\tau}\right\| e^{-\lambda\left(t-t_{m-1}-\tau\right)}, & t \in\left[t_{m-1}+\tau, t_{m}\right)\end{cases}
$$

and

$$
\begin{gathered}
\left\|x_{t_{m-1}+\tau}\right\| \leq M\left\|x_{t_{m-1}}\right\| e^{C M \tau e^{\beta \tau}}, \\
\left\|x_{t_{m}}\right\| \leq e^{(\alpha+\lambda) \tau} M\left\|x_{t_{m-1}+\tau}\right\| e^{-\lambda\left(t_{m}-t_{m-1}\right)} .
\end{gathered}
$$

Then, it follows from (3) and (4) that

$$
\left\|x_{t}\right\| \leq \begin{cases}e^{M_{m}}\|\varphi\| e^{-\lambda t_{m}} e^{\left|C M \tau e^{\beta \tau}-\beta\right| \tau+\beta \tau}, & t \in\left[t_{m}, t_{m}+\tau\right), \\ e^{M_{m}}\|\varphi\| e^{-\lambda t} e^{\alpha \tau}, & t \in\left[t_{m}+\tau, t_{m+1}\right) .\end{cases}
$$

In conclusion, for $t \in\left[t_{m}, t_{m+1}\right)$, where $m=1,2, \cdots$, we have

$$
\left\|x_{t}\right\| \leq \hat{M}\|\varphi\| e^{M_{m}-\lambda t_{m}} e^{-\lambda\left(t-t_{m}\right)}
$$

where $\hat{M} \in R_{+}$is a positive constant.

By (5), it can be shown that

(a) If the switching law satisfies $M_{m}-\lambda t_{m} \leq \tilde{M}, \quad m \in N_{+}$, then (5) implies $\left\|x_{t}\right\| \leq \hat{M}\|\varphi\| e^{\tilde{M}}, \quad t \geq 0$, meaning that (2) is SWUS;

(b) If the switching law satisfies $\lim _{m \rightarrow \infty}\left(M_{m}-\lambda t_{m}\right)=-\infty$, then (2) is SWUS and (5) implies $\lim _{t \rightarrow \infty}|x(t, 0, \varphi, \sigma)|=0$, for all $\varphi \in C_{\tau}$, meaning that (2) is GSWUS;

(c) If the switching law satisfies $\lim \sup _{m \rightarrow \infty} \frac{M_{m}-\lambda t_{m}}{t_{m}}<0$, then there exist $\varepsilon>0$ and $N \in N_{+}$such that for all $m \geq N, M_{m}-\lambda t_{m}<-\varepsilon t_{m}$, (without loss of generality, we may choose $\varepsilon \leq \lambda$ ). Thus, there exists a positive constant $\Pi$ such that $\left\|x_{t}\right\| \leq \Pi\|\varphi\| e^{-\varepsilon t}, t \geq 0$, meaning that (2) is GESWUS. 
Corollary 3.1. Suppose that the conditions of Theorem 3.1 hold. Then the switched control system (2) is:

(a) SWUS, if the switching law $\sigma$ satisfies

$$
t_{m}-t_{m-1} \geq \frac{2 \ln M+\tau\left(C M e^{\beta \tau}+\alpha+\tau\right)}{\lambda}, \quad m \in N_{+} ;
$$

(b) GASWUS, if the switching law $\sigma$ satisfies

$$
t_{m}-t_{m-1} \geq \frac{2 \ln M+\tau\left(C M e^{\beta \tau}+\alpha+\tau\right)}{\lambda}+\frac{1}{m}, \quad m \in N_{+} ;
$$

(c) GESWUS, if the switching law $\sigma$ satisfies

$$
\limsup _{m \rightarrow \infty} \frac{2 \ln M+\tau\left(C M e^{\beta \tau}+\alpha+\tau\right)}{t_{m}-t_{m-1}}=\lambda-\varepsilon,
$$

where $\varepsilon>0$ is a constant.

Proof. For $t \in\left[t_{m}, t_{m+1}\right)$, from (5), it follows that

$$
\begin{aligned}
\left\|x_{t}\right\| & \leq \hat{M}\|\varphi\| e^{M_{m}-\lambda t_{m}} e^{-\lambda\left(t-t_{m}\right)} \\
& \leq \hat{M}\|\varphi\| e^{\sum_{i=1}^{m}\left[2 \ln M+\tau\left(C M e^{\beta \tau}+\alpha+\tau\right)-\lambda\left(t_{i}-t_{i-1}\right)\right]} e^{-\lambda\left(t-t_{m}\right)} .
\end{aligned}
$$

Thus, it holds that

(a) If the switching law satisfies

$$
t_{m}-t_{m-1} \geq \frac{2 \ln M+\tau\left(C M e^{\beta \tau}+\alpha+\tau\right)}{\lambda}, \quad m \in N_{+},
$$

then

$$
\sum_{i=1}^{m}\left[2 \ln M+\tau\left(C M e^{\beta \tau}+\alpha+\tau\right)-\lambda\left(t_{i}-t_{i-1}\right)\right] \leq 0
$$

and

$$
\left\|x_{t}\right\| \leq \hat{M}\|\varphi\| \text {. }
$$

This implies that the switched control system (2) is SWUS. 
(b) If the switching law satisfies

$$
t_{m}-t_{m-1} \geq \frac{2 \ln M+\tau\left(C M e^{\beta \tau}+\alpha+\tau\right)}{\lambda}+\frac{1}{m}, \quad m \in N_{+},
$$

then

$$
\sum_{i=1}^{m}\left[2 \ln M+\tau\left(C M e^{\beta \tau}+\alpha+\tau\right)-\lambda\left(t_{i}-t_{i-1}\right)\right] \leq-\lambda \sum_{i=1}^{m} \frac{1}{i}
$$

and for all $\varphi \in C_{\tau}$,

$$
\lim _{t \rightarrow \infty}|x(t ; 0, \varphi, \sigma)|=0 .
$$

This means that the switched control system (2) is GASWUS.

(c) If the switching law satisfies

$$
\limsup _{m \rightarrow \infty} \frac{2 \ln M+\tau\left(C M e^{\beta \tau}+\alpha+\tau\right)}{t_{m}-t_{m-1}}=\lambda-\varepsilon,
$$

then there exists a $N \in N_{+}$, such that for $m \geq N$,

$$
\frac{2 \ln M+\tau\left(C M e^{\beta \tau}+\alpha+\tau\right)-\lambda\left(t_{m}-t_{m-1}\right)}{t_{m}-t_{m-1}} \leq-\frac{\varepsilon}{2},
$$

and thus

$$
\left\|x_{t}\right\| \leq \mathbb{M} \hat{M}\|\varphi\| e^{-\frac{\bar{\varepsilon}}{2} t}
$$

where $\mathbb{M}$ is a positive number. This implies that the switched control system (2) is GESWUS.

\section{Nonlinear Systems}

In this section, we consider the following nonlinear switched control system

$$
\dot{x}(t)=A_{\sigma(t)} x(t)+B_{\sigma(t)} u(t)+f_{\sigma(t)}\left(x(t), \int_{t-\tau_{1}}^{t} x(s) d s\right) .
$$

The aim is to design a switching law and a state feedback controller

$$
u(t)=K_{\gamma(t)} x(t), \quad t \geq 0,
$$


where $K_{\gamma(t)} \in R^{n \times n}$ and $\gamma(t)$ is the detection function of $\sigma(t)$, (i.e., $\gamma(t)=$ $\left.\sigma\left(t-\tau_{2}\right)\right)$, such that the closed-loop switched system

$$
\dot{x}(t)=A_{\sigma(t)} x(t)+B_{\sigma(t)} K_{\sigma\left(t-\tau_{2}\right)} x(t)+f_{\sigma(t)}\left(x(t), \int_{t-\tau_{1}}^{t} x(s) d s\right)
$$

is globally asymptotically stable or globally exponentially stable, where $\tau_{1}, \tau_{2} \geq$ 0 .

To continue, let $x\left(t ; t_{0}, \varphi\right)$ be the solution of the system,

$$
\dot{x}(t)=A x(t)+f\left(x(t), \int_{t-\tau}^{t} x(s) d s\right),
$$

with $x_{t_{0}}=\varphi, \varphi \in C_{\tau}$.

Lemma 4.1. Suppose that there exist positive numbers $\epsilon, \lambda_{1}, \lambda_{2}$ and matrices $E_{1}=E_{1}^{T}, E_{2} \in R^{n \times n}$, such that

$$
\begin{gathered}
2 x^{T}(t) f\left(x(t), \int_{t-\tau}^{t} x(s) d s\right) \leq x^{T}(t) E_{1} x(t)+\int_{t-\tau}^{t} x^{T}(t) E_{2} x(s) d s . \\
\lambda\left(A+A^{T}+E_{1}+\tau \epsilon^{-1} E\right) \leq-\lambda_{1}, \lambda\left(E_{2}{ }^{T} E_{2}\right) \leq \lambda_{2} .
\end{gathered}
$$

where $\lambda(\cdot)$ stands for the maximum eigenvalue of the corresponding matrix.

Then

(i) $\lambda_{1}>\tau \epsilon \lambda_{2} \geq 0$ implies

$$
|x(t)| \leq\|\varphi\| e^{-\lambda\left(t-t_{0}\right)}
$$

where $\lambda$ is the unique positive solution of the equation $2 \lambda=\lambda_{1}-$ $\tau \in \lambda_{2} e^{2 \lambda \tau}$.

(ii) $0 \leq \lambda_{1} \leq \tau \epsilon \lambda_{2}$ implies

$$
|x(t)| \leq\|\varphi\| e^{\frac{1}{2}\left(\tau \epsilon \lambda_{2} e^{\lambda_{1} \tau}-\lambda_{1}\right)\left(t-t_{0}\right)} .
$$


Proof. Let $V=x^{T} x$. Then, by Lemma 2.4, we have

$$
\begin{aligned}
\left.\dot{V}\right|_{(8)} & =x^{T}(t)\left(A+A^{T}\right) x(t)+2 x^{T}(t) f\left(x(t), \int_{t-\tau}^{t} x(s) d s\right) \\
& \leq x^{T}(t)\left(A+A^{T}\right) x(t)+x^{T}(t) E_{1} x(t)+\int_{t-\tau}^{t} x^{T}(t) E_{2} x(s) d s \\
& \leq x^{T}(t)\left(A+A^{T}+E_{1}\right) x(t)+\int_{t-\tau}^{t}\left[\epsilon^{-1} x^{T}(t) x(t)+\epsilon x^{T}(s) E_{2}^{T} E_{2} x(s)\right] d s \\
& \leq x^{T}\left(A+A^{T}+E_{1}+\tau \epsilon^{-1} E\right) x(t)+\epsilon \int_{t-\tau}^{t} x^{T}(s) E_{2}^{T} E_{2} x(s) d s \\
& \leq-\lambda_{1} V(t)+\tau \epsilon \lambda_{2}\left\|V_{t}\right\| .
\end{aligned}
$$

If $\lambda_{1}>\tau \epsilon \lambda_{2} \geq 0$, then it follows from Lemma 2.2 that

$$
V(t) \leq\left\|V_{t_{0}}\right\| e^{-2 \lambda\left(t-t_{0}\right)},
$$

which shows the validity of (9).

If $\lambda_{1} \leq \tau \epsilon \lambda_{2}$, then it follows from Lemma 2.3 that

$$
|V(t)| \leq\left\|V_{t_{0}}\right\| e^{\left(\tau \epsilon \lambda_{2} e^{\lambda_{1} \tau}-\lambda_{1}\right)\left(t-t_{0}\right)},
$$

and hence

$$
|x(t)| \leq\|\varphi\| e^{\frac{1}{2}\left(\tau \in \lambda_{2} e^{\lambda_{1} \tau}-\lambda_{1}\right)\left(t-t_{0}\right)} .
$$

Now, consider system (7) under the following assumptions.

\section{Assumption $\mathbb{A}_{2}$}

i) There exist positive numbers $\epsilon_{i}, \lambda_{1 i}, \lambda_{2 i}, \lambda_{3 i}$ and matrices $E_{1 i}=E_{1 i}^{T}, E_{2 i} \in$ $R^{n \times n}, i \in \Theta$, such that for all $i \in \Theta$

$$
2 x^{T}(t) f_{i}\left(x(t), \int_{t-\tau_{1}}^{t} x(s) d s\right) \leq x^{T}(t) E_{1 i} x(t)+\int_{t-\tau_{1}}^{t} x^{T}(t) E_{2 i} x(s) d s ;
$$

ii) $\lambda\left(\left(A_{i}+B_{i} K_{i}\right)^{T}+\left(A_{i}+B_{i} K_{i}\right)+E_{1 i}+\tau_{1} \epsilon_{i}^{-1} E\right) \leq-\lambda_{1 i}$,

$$
\begin{aligned}
& \lambda\left(\left(A_{i}+B_{i} K_{j}\right)^{T}+\left(A_{i}+B_{i} K_{j}\right)+E_{1 i}+\tau_{1} \epsilon_{i}^{-1} E\right) \leq-\lambda_{2 i}, \quad i \neq j, \\
& \lambda\left(E_{2 i}^{T} E_{2 i}\right) \leq \lambda_{3 i}, \quad i, j \in \Theta .
\end{aligned}
$$




\subsection{The case $\tau_{1} \geq \tau_{2}$}

Theorem 4.1. Suppose that Assumption $\mathbb{A}_{2}$ hold and that

(i) $\lambda_{1 i}-\tau_{1} \epsilon_{i} \lambda_{3 i}>0, \lambda_{(i)}$ is the unique positive solution of the equation $2 \lambda_{(i)}=\lambda_{1 i}-\tau_{1} \epsilon \lambda_{3 i} e^{2 \lambda_{(i)} \tau}$ and $\lambda=\min _{i \in \Theta}\left\{\lambda_{(i)}\right\}$;

(ii) $\lambda_{2 i}-\tau_{1} \epsilon_{i} \lambda_{3 i}>0, \hat{\lambda}_{(i)}$ is the unique positive solution of the equation $2 \hat{\lambda}_{(i)}=\lambda_{2 i}-\tau_{1} \epsilon \lambda_{3 i} e^{2 \hat{\lambda}_{(i)} \tau}$ and $\hat{\lambda}=\min _{i \in \Theta}\left\{\hat{\lambda}_{(i)}\right\}$; and

(iii) the switching law $\sigma=\left\{\left(i_{1}, t_{1}\right), \cdots,\left(i_{k}, t_{k}\right), \cdots\right\}$ satisfies $t_{k}-t_{k-1} \geq$ $\tau_{1}+\tau_{2}$.

Then, the switched control system (7) is GESWUS.

Proof. Let $\sigma(t)$ be a given switching signal. For the case when $t \in\left[0, t_{1}\right)$, the $i_{1}^{\text {th }}$ subsystem is active, i.e.,

$$
\dot{x}(t)=A_{i_{1}} x(t)+B_{i_{1}} K_{i_{1}} x(t)+f_{i_{1}}\left(x(t), \int_{t-\tau_{1}}^{t} x(s) d s\right),
$$

Then, by the conditions of the theorem and Lemma 4.1(i), it follows that

$$
|x(t)| \leq\|\varphi\| e^{-\lambda t}
$$

We claim that, for any $m \in N_{+}$and $t \in\left[t_{m}, t_{m+1}\right)$, the solution of system (7) satisfies:

$$
\begin{aligned}
& (*)_{1}:|x(t)| \leq\|\varphi\| e^{-\lambda\left(t_{m}-m \tau_{1}\right)} e^{-\hat{\lambda}\left(t-t_{m}\right)}, \quad t \in\left[t_{m}, t_{m}+\tau_{2}\right), \\
& (*)_{2}: \quad|x(t)| \leq\|\varphi\| e^{-\lambda\left(t-(m+1) \tau_{1}\right)}, \quad t \in\left[t_{m}+\tau_{2}, t_{m+1}\right) .
\end{aligned}
$$

Indeed, when $t \in\left[t_{1}, t_{1}+\tau_{2}\right)$, the active subsystem is:

$$
\dot{x}(t)=A_{i_{2}} x(t)+B_{i_{2}} K_{i_{1}} x(t)+f_{i_{2}}\left(x(t), \int_{t-\tau_{1}}^{t} x(s) d s\right) .
$$

Thus, by the conditions of theorem and Lemma 4.1(i), we obtain

$$
|x(t)| \leq\left\|x_{t_{1}}\right\| e^{-\hat{\lambda}\left(t-t_{1}\right)} \leq\|\varphi\| e^{-\lambda\left(t_{1}-\tau_{1}\right)} e^{-\hat{\lambda}\left(t-t_{1}\right)}, \quad t \in\left[t_{1}, t_{1}+\tau_{2}\right) .
$$


For $t \in\left[t_{1}+\tau_{2}, t_{2}\right)$, we have

$$
\begin{aligned}
|x(t)| & \leq\left\|x_{t_{1}+\tau_{2}}\right\|_{\tau} e^{-\lambda\left(t-t_{1}-\tau_{2}\right)} \\
& =\max \left\{\sup _{t_{1}+\tau_{2}-\tau_{1} \leq \theta \leq t_{1}}|x(\theta)|, \sup _{t_{1} \leq \theta \leq t_{1}+\tau_{2}}|x(\theta)|\right\} e^{-\lambda\left(t-t_{1}-\tau_{2}\right)} \\
& \leq \max \left\{\|\varphi\| e^{-\lambda\left(t_{1}+\tau_{2}-\tau_{1}\right)},\left\|x_{t_{1}}\right\|\right\} e^{-\lambda\left(t-t_{1}-\tau_{2}\right)} \\
& \leq \max \left\{\|\varphi\| e^{-\lambda\left(t_{1}+\tau_{2}-\tau_{1}\right)},\|\varphi\| e^{-\lambda\left(t_{1}-\tau_{1}\right)}\right\} e^{-\lambda\left(t-t_{1}-\tau_{2}\right)} \\
& =\|\varphi\| e^{-\lambda\left(t-\tau_{2}-\tau_{1}\right)} \leq\|\varphi\| e^{-\lambda\left(t-2 \tau_{1}\right)}
\end{aligned}
$$

It means that $(*)_{1}$ and $(*)_{2}$ hold for $m=1$.

Suppose that $(*)_{1}$ and $(*)_{2}$ hold for $m \in N_{+}$and we will show that $(*)_{1}$ and $(*)_{2}$ hold for $m+1$.

For $t \in\left[t_{m+1}, t_{m+1}+\tau_{2}\right)$, we have

$$
\begin{aligned}
|x(t)| & \leq\left\|x_{t_{m+1}}\right\| e^{-\hat{\lambda}\left(t-t_{m+1}\right)} \\
& \leq\left\|x_{0}\right\| e^{-\lambda\left(t_{m+1}-(m+1) \tau_{1}\right)} e^{-\hat{\lambda}\left(t-t_{m+1}\right)} .
\end{aligned}
$$

On this basis, it follows that for $t \in\left[t_{m+1}+\tau_{2}, t_{m+2}\right)$,

$$
\begin{aligned}
|x(t)| & \leq\left\|x_{t_{m+1}+\tau_{2}}\right\| e^{-\lambda\left(t-t_{m+1}-\tau_{2}\right)} \\
& \leq \max \left\{\sup _{t_{m+1}+\tau_{2}-\tau_{1} \leq \theta \leq t_{m+1}}|x(\theta)|, \sup _{t_{m+1} \leq \theta \leq t_{m+1}+\tau_{2}}|x(\theta)|\right\} e^{-\lambda\left(t-t_{m+1}-\tau_{2}\right)} \\
& \leq \max \left\{\|\varphi\| e^{-\lambda\left(t_{m+1}+\tau_{2}-(m+2) \tau_{1}\right)},\|\varphi\| e^{-\lambda\left(t_{m+1}-(m+1) \tau_{1}\right)}\right\} e^{-\lambda\left(t-t_{m+1}-\tau_{2}\right)} \\
& =\|\varphi\| e^{-\lambda\left(t-(m+2) \tau_{1}\right)} .
\end{aligned}
$$

This means that $(*)_{1}$ and $(*)_{2}$ hold for $m+1$. By mathematical induction principle, we conclude that for all $m \in N_{+},(*)_{1}$ and $(*)_{2}$ hold.

Now, from $(*)_{1}$ and $(*)_{2}$, we have

$$
|x(t)| \leq\|\varphi\| e^{\lambda \tau_{1}} e^{-\lambda\left(t-m \tau_{1}\right)}, \quad t \in\left[t_{m}, t_{m+1}\right) .
$$

Since the switching law satisfies $t_{k}-t_{k-1} \geq \tau_{1}+\tau_{2}$, thus

$$
\liminf _{k \rightarrow \infty} \frac{t_{k}-k \tau_{1}}{t_{k}}>0
$$


so there exist $\varepsilon>0$ and $N \in N_{+}$, such that for $k \geq N, t_{k}-k \tau_{1}>\varepsilon t_{k}$. Thus

$$
-\lambda\left(t_{k}-k \tau_{1}\right)<-\lambda \varepsilon t_{k}, k \geq N
$$

and hence

$$
\begin{aligned}
|x(t)| & \leq\|\varphi\| e^{\lambda \tau_{1}} e^{-\lambda\left(t-m \tau_{1}\right)} \\
& \leq\|\varphi\| e^{\lambda \tau_{1}} e^{-\lambda\left(t_{m}-m \tau_{1}\right)} e^{-\lambda\left(t-t_{m}\right)} \\
& \leq\|\varphi\| e^{\lambda \tau_{1}} e^{-\lambda \varepsilon t_{m}} e^{-\lambda\left(t-t_{m}\right)}, \quad m \geq N
\end{aligned}
$$

Therefore, the conclusion of the theorem follows readily.

Corollary 4.1. Suppose that the conditions of Theorem 4.1 hold and that $\tau_{2}=$ 0 . Then, the switched control system (7) is:

a) SWUS, if the switching law $\sigma$ satisfies $\lim _{k \rightarrow \infty}\left(t_{k}-k \tau_{1}\right)<\infty$;

b) GASWUS, if the switching law $\sigma$ satisfies $\lim _{k \rightarrow \infty}\left(t_{k}-k \tau_{1}\right)=\infty$;

c) GESWUS, if the switching law $\sigma$ satisfies $\liminf _{k \rightarrow \infty} \frac{t_{k}-k \tau_{1}}{t_{k}}>0$.

Corollary 4.2. Suppose that the conditions of Theorem 4.1 hold. Then, the switched control system (7) is GESWUS, if the switching law $\sigma$ satisfies

$$
\liminf _{m \rightarrow \infty} \frac{t_{m}-t_{m-1}-\tau_{1}}{t_{m}-t_{m-1}}=\varepsilon>0 .
$$

Proof. From (11), it follows that

$$
\begin{aligned}
|x(t)| & \leq\|\varphi\| e^{\lambda \tau_{1}} e^{-\lambda\left(t-m \tau_{1}\right)} \\
& \leq\|\varphi\| e^{-\sum_{i=1}^{m} \lambda\left(t_{i}-t_{i-1}-\tau_{1}\right)} e^{-\lambda\left(t-t_{m}-\tau_{1}\right)} .
\end{aligned}
$$

Since the switching law satisfies

$$
\liminf _{m \rightarrow \infty} \frac{t_{m}-t_{m-1}-\tau_{1}}{t_{m}-t_{m-1}}=\varepsilon,
$$

there exists an $N \in N_{+}$, such that for $m \geq N$ and $\lambda>0$,

$$
-\lambda \frac{t_{m}-t_{m-1}-\tau_{1}}{t_{m}-t_{m-1}} \leq-\frac{\varepsilon}{2} \lambda
$$


and hence

$$
\begin{aligned}
\left\|x_{t}\right\| & \leq\|\varphi\| e^{-\lambda \sum_{i=1}^{m}\left(t_{i}-t_{i-1}-\tau_{1}\right)} e^{-\lambda\left(t-t_{m}-\tau_{1}\right)} \\
& \leq\|\varphi\| e^{-\lambda \sum_{i=1}^{N}\left(t_{i}-t_{i-1}-\tau_{1}\right)} e^{-\lambda \sum_{i=N}^{m}\left(t_{i}-t_{i-1}-\tau_{1}\right)} e^{-\lambda\left(t-t_{m}-\tau_{1}\right)} \\
& \leq\|\varphi\| e^{-\lambda \sum_{i=1}^{N}\left(t_{i}-t_{i-1}-\tau_{1}\right)} e^{-\frac{\varepsilon}{2} \lambda\left(t_{m}-t_{N}\right)} e^{-\lambda\left(t-t_{m}-\tau_{1}\right)} \\
& \leq \mathbb{M}\|\varphi\| e^{-\frac{\varepsilon}{2} \lambda t}
\end{aligned}
$$

where $\mathbb{M}$ is a positive number. Thus the switched system is GESWUS.

Using the similar proof method of Theorem 4.1 and Lemma 4.1(ii), we can prove the following results.

Theorem 4.2. Suppose that Assumption $\mathbb{A}_{2}$ holds and that

(i) $\lambda_{1 i}-\tau_{1} \epsilon_{i} \lambda_{3 i}>0$ and $\lambda_{(i)}$ is the unique positive solution of the equation $2 \lambda_{(i)}=\lambda_{1 i}-\tau_{1} \epsilon \lambda_{3 i} e^{2 \lambda_{(i)} \tau}$ and $\lambda=\min _{i \in \Theta}\left\{\lambda_{(i)}\right\}$;

(ii) $\lambda_{2 i}-\tau_{1} \epsilon_{i} \lambda_{3 i} \leq 0, i \in \Theta$; and

(iii) the switching law $\sigma=\left\{\left(i_{1}, t_{1}\right), \cdots,\left(i_{k}, t_{k}\right), \cdots\right\}$ satisfies $t_{k}-t_{k-1} \geq$ $\tau_{1}+\tau_{2}$.

Then, the switched control system (7) is:

a) SWUS, if there exists $M \in R$, such that for all $m \in N_{+}, m\left(\Delta+\lambda \tau_{2}\right)-$ $\lambda\left(t-m \tau_{1}\right) \leq M$

b) GASWUS, if $\lim _{m \rightarrow \infty}\left[m\left(\Delta+\lambda \tau_{2}\right)-\lambda\left(t-m \tau_{1}\right)\right]=-\infty$; and

c) GESWUS, if $\liminf _{k \rightarrow \infty} \frac{m\left(\Delta+\lambda \tau_{2}\right)-\lambda\left(t-m \tau_{1}\right)}{t}<0$,

where $2 \Delta=\max _{i \in \Theta}\left\{\left(\lambda_{3 i} \epsilon_{i} \tau_{1} e^{\lambda_{2 i} \tau_{1}}-\lambda_{2 i}\right) \tau_{2}\right\}$.

Corollary 4.3. Suppose that the conditions of Theorem 4.2 hold. Then, the switched control system (7) is:

a) $S W U S$, if there exists $M>0$ such that $\sum_{k=1}^{m}\left(t_{k}-t_{k-1}-\tau_{1}-\tau_{2}-\frac{\Delta}{\lambda}\right) \geq$ $-M$

b) GASWUS, if $\lim _{k \rightarrow \infty} \sum_{k=1}^{m}\left(t_{k}-t_{k-1}-\tau_{1}-\tau_{2}-\frac{\Delta}{\lambda}\right)=+\infty$; and

c) GESWUS, if $\liminf \operatorname{in}_{k \rightarrow \infty} \frac{\sum_{k=1}^{m}\left(t_{k}-t_{k-1}-\tau_{1}-\tau_{2}-\frac{\Delta}{\lambda}\right)}{t}>0$,

where $2 \Delta=\max _{i \in \Theta}\left\{\left(\lambda_{3 i} \epsilon_{i} \tau_{1} e^{\lambda_{2 i} \tau_{1}}-\lambda_{2 i}\right) \tau_{2}\right\}$. 


\subsection{The case of $\tau_{2} \geq \tau_{1}$}

To begin, we notice that since $\tau_{2} \geq \tau_{1},\left\|x_{t}\right\|=\sup _{t-\tau_{2} \leq \theta \leq t}|x(\theta)|$.

By similar argument, we can prove following results.

Theorem 4.3. Suppose that Assumption $\mathbb{A}_{2}$ holds and that

(i) $\lambda_{1 i}-\tau_{1} \epsilon_{i} \lambda_{3 i}>0$ and $\lambda_{(i)}$ is the unique positive solution of the equation $2 \lambda_{(i)}=\lambda_{1 i}-\tau_{1} \epsilon_{i} \lambda_{3 i} e^{2 \lambda_{(i)} \tau}$ and $\lambda=\min _{i \in \Theta}\left\{\lambda_{(i)}\right\} ;$ and

(ii) $\lambda_{2 i}-\tau_{1} \epsilon_{i} \lambda_{3 i}>0$ and $\hat{\lambda}_{(i)}$ is the unique positive solution of the equation $2 \hat{\lambda}_{(i)}=\lambda_{2 i}-\tau_{1} \epsilon_{i} \lambda_{3 i} e^{2 \hat{\lambda}_{(i)} \tau}$ and $\hat{\lambda}=\min _{i \in \Theta}\left\{\hat{\lambda}_{(i)}\right\}$.

Then, the switched control system (7) is:

a) SWUS, if the switching law $\sigma=\left\{\left(i_{1}, t_{1}\right), \cdots,\left(i_{m}, t_{m}\right), \cdots\right\}$ satisfies $\tau_{2} \leq t_{m}-t_{m-1} \leq 2 \tau_{2}$, for $m \in N_{+} ;$

b) GASWUS, if the switching law $\sigma=\left\{\left(i_{1}, t_{1}\right), \cdots,\left(i_{m}, t_{m}\right), \cdots\right\}$ satisfies $t_{m}-t_{m-1} \geq 2 \tau_{2}$ for $m \in N_{+}$and $\lim _{m \rightarrow \infty}\left(t_{m}-2 m \tau_{2}\right)=\infty$; and

c) GESWUS, if the switching law $\sigma=\left\{\left(i_{1}, t_{1}\right), \cdots,\left(i_{m}, t_{m}\right), \cdots\right\}$ satisfies $t_{m}-t_{m-1} \geq 2 \tau_{2}, m \in N_{+}$and $\liminf _{m \rightarrow \infty} \frac{t_{m}-2 m \tau_{2}}{t_{m}}>0$.

Proof. Let $\sigma=\left\{\left(i_{1}, t_{1}\right), \cdots,\left(i_{m}, t_{m}\right), \cdots\right\}$ be a given a switching signal. For $t \in\left[0, t_{1}\right)$, the $i_{1}^{\text {th }}$ subsystem is active, i.e.,

$$
\dot{x}(t)=A_{i_{1}} x(t)+B_{i_{1}} K_{i_{1}} x(t)+f_{i_{1}}\left(x(t), \int_{t-\tau_{1}}^{t} x(s) d s\right),
$$

Thus, by the conditions of the theorem and Lemma 4.1(i), it is clear that

$$
|x(t)| \leq\|\varphi\| e^{-\lambda t} .
$$

a) We claim that, for any $m \in N_{+}$, the solution of system (7) satisfies:

$$
\begin{aligned}
& (*)_{3}:|x(t)| \leq\|\varphi\| e^{-\lambda\left(t_{1}-\tau_{2}\right)} e^{-\hat{\lambda}\left(t-t_{m}\right)}, \quad t \in\left[t_{m}, t_{m}+\tau_{2}\right), \\
& (*)_{4}: \quad|x(t)| \leq\|\varphi\| e^{-\lambda\left(t_{1}-2 \tau_{2}\right)} e^{-\lambda\left(t-t_{m}\right)}, \quad t \in\left[t_{m}+\tau_{2}, t_{m+1}\right) .
\end{aligned}
$$

Indeed, for $t \in\left[t_{1}, t_{1}+\tau_{2}\right)$, the active subsystem is:

$$
\dot{x}(t)=A_{i_{2}} x(t)+B_{i_{2}} K_{i_{1}} x(t)+f_{i_{2}}\left(x(t), \int_{t-\tau_{1}}^{t} x(s) d s\right),
$$


Thus, by the conditions of the theorem and Lemma 4.1(i), we obtain

$$
|x(t)| \leq\left\|x_{t_{1}}\right\| e^{-\hat{\lambda}\left(t-t_{1}\right)}=\|\varphi\| e^{-\lambda\left(t_{1}-\tau_{2}\right)} e^{-\hat{\lambda}\left(t-t_{1}\right)} .
$$

Now, for $t \in\left[t_{1}+\tau_{2}, t_{2}\right)$, we have

$$
\begin{aligned}
|x(t)| & \leq\left\|x_{t_{1}+\tau_{2}}\right\| e^{-\lambda\left(t-t_{1}-\tau_{2}\right)} \\
& =\sup _{t_{1} \leq \theta \leq t_{1}+\tau_{2}}\|\varphi\| e^{-\lambda\left(t_{1}-\tau_{2}\right)} e^{-\hat{\lambda}\left(\theta-t_{1}\right)} e^{-\lambda\left(t-t_{1}-\tau_{2}\right)} \\
& =\|\varphi\| e^{-\lambda\left(t_{1}-2 \tau_{2}\right)} e^{-\lambda\left(t-t_{1}\right)} .
\end{aligned}
$$

It means that $(*)_{3}$ and $(*)_{4}$ hold for $m=1$.

Suppose that $(*)_{3}$ and $(*)_{4}$ hold for $m \in N_{+}$and we shall show prove that $(*)_{3}$ and $(*)_{4}$ hold for $m+1$.

For $t \in\left[t_{m+1}, t_{m+1}+\tau_{2}\right)$, we have

$$
\begin{aligned}
|x(t)| \leq & \left\|x_{t_{m+1}}\right\| e^{-\hat{\lambda}\left(t-t_{m+1}\right)} \\
= & \sup _{t_{m+1}-\tau_{2} \leq \theta \leq t_{m+1}}\|x(\theta)\| e^{-\hat{\lambda}\left(t-t_{m+1}\right)} \\
= & \max \left\{\sup _{t_{m+1}-\tau_{2} \leq \theta \leq t_{m}+\tau_{2}}\|x(\theta)\|, \sup _{t_{m}+\tau_{2} \leq \theta \leq t_{m+1}}\|x(\theta)\|\right\} e^{-\hat{\lambda}\left(t-t_{m+1}\right)} \\
= & \max \left\{\sup _{t_{m+1}-\tau_{2} \leq \theta \leq t_{m}+\tau_{2}}\|\varphi\| e^{-\lambda\left(t_{1}-\tau_{2}\right)} e^{-\hat{\lambda}\left(\theta-t_{m}\right)},\right. \\
& \left.\sup _{t_{m}+\tau_{2} \leq \theta \leq t_{m+1}}\|\varphi\| e^{-\lambda\left(t_{1}-2 \tau_{2}\right)} e^{-\lambda\left(\theta-t_{m}\right)}\right\} e^{-\hat{\lambda}\left(t-t_{m+1}\right)} \\
\leq & \max \left\{\|\varphi\| e^{-\lambda\left(t_{1}-\tau_{2}\right)} e^{-\hat{\lambda}\left(t_{m+1}-\tau_{2}-t_{m}\right)},\|\varphi\| e^{-\lambda\left(t_{1}-\tau_{2}\right)}\right\} e^{-\hat{\lambda}\left(t-t_{m+1}\right)} \\
\leq & \|\varphi\| e^{-\lambda\left(t_{1}-\tau_{2}\right)} e^{-\hat{\lambda}\left(t-t_{m+1}\right)}
\end{aligned}
$$

Now, for $t \in\left[t_{m+1}+\tau_{2}, t_{m+2}\right)$, we obtain

$$
\begin{aligned}
|x(t)| & \leq\left\|x_{t_{m+1}+\tau_{2}}\right\| e^{-\lambda\left(t-t_{m+1}-\tau_{2}\right)} \\
& =\sup _{t_{m+1} \leq \theta \leq t_{m+1}+\tau_{2}}\|\varphi\| e^{-\lambda\left(t_{1}-\tau_{2}\right)} e^{-\hat{\lambda}\left(\theta-t_{m+1}\right)} e^{-\lambda\left(t-t_{m+1}-\tau_{2}\right)} \\
& \leq\|\varphi\| e^{-\lambda\left(t_{1}-\tau_{2}\right)} e^{-\lambda\left(t-t_{m+1}-\tau_{2}\right)} \\
& \leq\|\varphi\| e^{-\lambda\left(t_{1}-2 \tau_{2}\right)} e^{-\lambda\left(t-t_{m+1}\right)}
\end{aligned}
$$


This means that for $m+1,(*)_{3}$ and $(*)_{4}$ hold. Thus, by mathematical induction principle, it is clear that for all $m \in N_{+},(*)_{3}$ and $(*)_{4}$ hold. From $(*)_{3}$ and $(*)_{4}$, it follows that

$$
|x(t)| \leq\|\varphi\| e^{-\lambda\left(t_{1}-\tau_{2}\right)}, \quad t \in\left[t_{m}, t_{m+1}\right),
$$

which implies that the the switched control system (7) is SWUS.

b) In this case, the switching law $\sigma$ satisfies the condition $t_{m}-t_{m-1} \geq 2 \tau_{2}$. We claim that, for any $m \in N_{+}$, the solution of system (7) satisfies:

$$
\begin{array}{ll}
(*)_{3_{1}}: & |x(t)| \leq\|\varphi\| e^{-\lambda\left(t_{m}-2 m \tau_{2}+\tau_{2}\right)} e^{-\hat{\lambda}\left(t-t_{m}\right)}, \quad t \in\left[t_{m}, t_{m}+\tau_{2}\right), \\
(*)_{4_{1}}: & |x(t)| \leq\|\varphi\| e^{-\lambda\left(t-2 m \tau_{2}\right)}, \quad t \in\left[t_{m}+\tau_{2}, t_{m+1}\right) .
\end{array}
$$

Indeed, for $t \in\left[t_{1}, t_{1}+\tau_{2}\right)$, the active subsystem is:

$$
\dot{x}(t)=A_{i_{2}} x(t)+B_{i_{2}} K_{i_{1}} x(t)+f_{i_{2}}\left(x(t), \int_{t-\tau_{1}}^{t} x(s) d s\right),
$$

Thus, by the conditions of the theorem and Lemma 4.1(i), it follows that

$$
|x(t)| \leq\left\|x_{t_{1}}\right\| e^{-\hat{\lambda}\left(t-t_{1}\right)} \leq\|\varphi\| e^{-\lambda\left(t_{1}-\tau_{2}\right)} e^{-\hat{\lambda}\left(t-t_{1}\right)} .
$$

Now, for $t \in\left[t_{1}+\tau_{2}, t_{2}\right)$, we have

$$
\begin{aligned}
|x(t)| & \leq\left\|x_{t_{1}+\tau_{2}}\right\| e^{-\lambda\left(t-t_{1}-\tau_{2}\right)} \\
& =\sup _{t_{1} \leq \theta \leq t_{1}+\tau_{2}}\|\varphi\| e^{-\lambda\left(t_{1}-\tau_{2}\right)} e^{-\hat{\lambda}\left(\theta-t_{1}\right)} e^{-\lambda\left(t-t_{1}-\tau_{2}\right)} \\
& \leq\|\varphi\| e^{-\lambda\left(t_{1}-\tau_{2}\right)} e^{-\lambda\left(t-t_{1}-\tau_{2}\right)}=\|\varphi\| e^{-\lambda\left(t-2 \tau_{2}\right)} .
\end{aligned}
$$

It means that $(*)_{3_{1}}$ and $(*)_{4_{1}}$ hold for $m=1$.

Suppose that $(*)_{3_{1}}$ and $(*)_{4_{1}}$ hold for $m \in N_{+}$and we shall show that $(*)_{31}$ and $(*)_{4_{1}}$ hold for $m+1$. 
For $t \in\left[t_{m+1}, t_{m+1}+\tau_{2}\right)$, we have

$$
\begin{aligned}
|x(t)| & \leq\left\|x_{t_{m+1}}\right\| e^{-\hat{\lambda}\left(t-t_{m+1}\right)} \\
& =\sup _{t_{m+1}-\tau_{2} \leq \theta \leq t_{m+1}}\|x(\theta)\| e^{-\hat{\lambda}\left(t-t_{m+1}\right)} \\
& \leq \sup _{t_{m+1}-\tau_{2} \leq \theta \leq t_{m+1}}\|\varphi\| e^{-\lambda\left(\theta-2 m \tau_{2}\right)} e^{-\hat{\lambda}\left(t-t_{m+1}\right)} \\
& \leq\|\varphi\| e^{-\lambda\left(t_{m+1}-2(m+1) \tau_{2}+\tau_{2}\right)} e^{-\hat{\lambda}\left(t-t_{m+1}\right)} .
\end{aligned}
$$

Now, for $t \in\left[t_{m+1}+\tau_{2}, t_{m+2}\right)$, it follows that

$$
\begin{aligned}
|x(t)| & \leq\left\|x_{t_{m+1}+\tau_{2}}\right\| e^{-\lambda\left(t-t_{m+1}-\tau_{2}\right)} \\
& =\sup _{t_{m+1} \leq \theta \leq t_{m+1}+\tau_{2}}\|\varphi\| e^{-\lambda\left(t_{m+1}-2(m+1) \tau_{2}+\tau_{2}\right)} e^{-\hat{\lambda}\left(\theta-t_{m+1}\right)} e^{-\lambda\left(t-t_{m+1}-\tau_{2}\right)} \\
& \leq\|\varphi\| e^{-\lambda\left(t_{m+1}-2(m+1) \tau_{2}+\tau_{2}\right)} e^{-\lambda\left(t-t_{m+1}-\tau_{2}\right)} \\
& \leq\|\varphi\| e^{-\lambda\left(t-2(m+1) \tau_{2}\right)} .
\end{aligned}
$$

This means that for $m+1,(*)_{3_{1}}$ and $(*)_{4_{1}}$ hold. Therefore, by mathematical induction principle, it is clear that for all $m \in N_{+},(*)_{3_{1}}$ and $(*)_{4}$ hold.

From $(*)_{3_{1}}$ and $(*)_{4_{1}}$, we can show that

$$
|x(t)| \leq\|\varphi\| e^{-\lambda\left(t-2 m \tau_{2}\right)}, \quad t \in\left[t_{m}, t_{m+1}\right),
$$

which implies that the switched control system (7) is GASWUS.

c) In this case, the switching law $\sigma$ satisfies the condition $t_{m}-t_{m-1} \geq 2 \tau_{2}$. It follows from (12) that

$$
|x(t)| \leq\|\varphi\| e^{-\lambda\left(t-2 m \tau_{2}\right)}, \quad t \in\left[t_{m}, t_{m+1}\right) .
$$

If

$$
\liminf _{k \rightarrow \infty} \frac{t_{m}-2 m \tau_{2}}{t_{m}}>0,
$$

then there exist $\varepsilon>0$ and $N \in N_{+}$, such that for all $m>N$, have

$$
\frac{t_{m}-2 m \tau_{2}}{t_{m}} \geq \frac{\varepsilon}{\lambda} \Rightarrow-\lambda\left(t_{m}-2 m \tau_{2}\right) \leq-\varepsilon t_{m}
$$


and hence (without loss of generality, we may choose $\varepsilon \leq \lambda$ )

$$
\begin{aligned}
|x(t)| & \leq\|\varphi\| e^{-\lambda\left(t-2 m \tau_{2}\right)}=\|\varphi\| e^{-\lambda\left(t_{m}-2 m \tau_{2}\right)} e^{-\lambda\left(t-t_{m}\right)} \\
& \leq\|\varphi\| e^{-\varepsilon t_{m}} e^{-\varepsilon\left(t-t_{m}\right)}=\|\varphi\| e^{-\varepsilon t} .
\end{aligned}
$$

Thus, the switched control system (7) is GESWUS.

Corollary 4.4. Suppose that the conditions of Theorem 4.3 hold. Then, the switched control system (7) is:

(a) GASWUS, if the switching law $\sigma$ satisfies $t_{m}-t_{m-1} \geq 2 \tau_{2}+\frac{1}{m}, \quad m \in$ $N_{+} ;$and

(b) GESWUS, if the switching law $\sigma$ satisfies

$$
\frac{t_{m}-t_{m-1}-2 \tau_{2}}{t_{m}-t_{m-1}} \geq \varepsilon>0 .
$$

Using the similar proof method of Theorem 4.3 and Lemma 4.1(ii), we can prove the following result:

Theorem 4.4. Suppose that Assumption $\mathbb{A}_{2}$ holds and that

(i) $\lambda_{1 i}-\tau_{1} \epsilon_{i} \lambda_{3 i}>0$ and $\lambda_{(i)}$ is the unique positive solution of the equation $2 \lambda_{(i)}=\lambda_{1 i}-\tau_{1} \epsilon_{i} \lambda_{3 i} e^{-2 \lambda_{(i)} \tau}$ and $\lambda=\min _{i \in \Theta}\left\{\lambda_{(i)}\right\}$;

(ii) $\lambda_{2 i}-\tau_{1} \epsilon_{i} \lambda_{3 i} \leq 0, i \in \Theta$; and

(iii) Switching law $\sigma=\left\{\left(i_{1}, t_{1}\right), \cdots,\left(i_{k}, t_{k}\right), \cdots\right\}$ satisfies $t_{k}-t_{k-1} \geq 2 \tau_{2}$.

Then, the switched control system (7) is:

a) $S W U S$, if there exists a $M>0$ such that for all $m \in N_{+},-\lambda t_{m}+$ $m(2 \lambda+\Delta) \tau_{2}<M$

b) GASWUS, if $\lim _{m \rightarrow \infty}-\lambda t_{m}+m(2 \lambda+\Delta) \tau_{2}=-\infty$;

c) GESWUS, if $\liminf _{t \rightarrow \infty} \frac{-\lambda t_{m}+m(2 \lambda+\Delta) \tau_{2}}{t_{m}}<0, t \in\left[t_{m}, t_{m+1}\right)$, where $2 \Delta=\max _{i \in \Theta}\left\{\left(\lambda_{3 i} \epsilon_{i} \tau_{1} e^{\lambda_{2 i} \tau_{1}}-\lambda_{2 i}\right) \tau_{2}\right\}$. 
Corollary 4.5. Suppose that the conditions of Theorem 4.4 hold. Then, the switched control system (7) is:

(a) SWUS, if the switching law $\sigma$ satisfies

$$
\lambda\left(t_{m}-t_{m-1}\right)-(2 \lambda+\Delta) \tau_{2} \geq 0, \quad m \in N_{+}
$$

(b) GASWUS, if the switching law $\sigma$ satisfies

$$
\lambda\left(t_{m}-t_{m-1}\right) \geq(2 \lambda+\Delta) \tau_{2}+\frac{1}{m}, \quad m \in N_{+} ;
$$

(c) GESWUS, if there exist $\varepsilon>0, N \in N_{+}$such that for all $m \geq N$, the switching law $\sigma$ satisfies

$$
\frac{\lambda\left(t_{m}-t_{m-1}\right)-(2 \lambda+\Delta) \tau_{2}}{t_{m}-t_{m-1}} \geq \varepsilon
$$

\section{Conclusion}

In this paper, we study the stabilization problem of switched control systems with switching signal time delay. New concepts of globally asymptotical or exponential stabilizability under state feedback controllers and switching laws are presented. Then, by using the method of Lyapunov functions and delay inequalities, appropriate state feedback controllers and switching laws are devised under which the resulting closed-loop switched systems are globally asymptotically stable and exponentially stable.

\section{Acknowledgements}

The research is partly supported by the grants $(11171079,11071257$ and 11371371) from National Science Funds of China, and a Discovery Grant from the Australian Research council.

\section{References}

[1] Branicky, M. S., Studies in hybrid systems: modeling, analysis, control, ScD Thesis. MIT EECS, 1995. 
[2] Li, Zhengguo, Soh, Yengchai, and Wen, Changyun, Switched and Impulsive Systems, Springer-Verlag, Berlin Heidelberg, 2005.

[3] Seborg, D.E., Edgar, T.F., Mellichamp, D.A. Process dynamics and control, JohnWiley and Sons, New York, 1989.

[4] Benzaouia, A., Saturated Switching Systems, Springer-Verlag London, 2012.

[5] Wick, M.A., Peleties, P., and Decarlo, R.A., Switched controller synthesis for the quadratic stabilization of a pair of unstable linear systems, European Journal of Control, 4 (1998), 140-147.

[6] Shorten, R.N., and Naredra, K.S., On the stability and existence of common Lyapunov functions for stable linear switching systems, Proccedings of IEEE Conference on Decision \& Control, 4 (1998), 3723-3724.

[7] Liberzon, D., Hespanha, J. P., and Morse, A.S., Stability of switched systems: a Lie-algebraic condition, Systems \&Control Letters, 37(3) (1999), 117-122.

[8] Xu, H., and Teo, K.L., Exponential stability with $l_{2}$-gain condition of nonlinear impulsive switched systems, IEEE Transaction on Automatic Control, 55 (2010), 2429-2433.

[9] Mahmoud, M. S., Switched Time-Delay Systems, Springer Science+Business Media, LLC, 2010.

[10] Liu, X., Stabilization of switched linear systems with mode-dependent time-varying delays, Applied Mathematics and Computation, 216(9) (2010), 2581-2586.

[11] Michael, S. Branicky, Multiple Lyapunov functions and other analysis tools for switched and hybrid systems, IEEE Transaction on Automatic Control, 43(4) (1998), 475-482.

[12] Liberzon, D., Switching in systems and control, Birkhäuser, 2003.

[13] Dayawansa, W. P. and Martin, C.F., A converse Lyapunov theorem for a class of dynamical systems which undergo switching, IEEE Transaction on Automatic Control, 44 (1999), 751-760. 
[14] Zhang, Y., Liu, X.and Shen, X.,Stability of switched systems with time delay Nonlinear Analysis: Hybrid Systems, Vol. 1(1), (2007), 44-58.

[15] Sun, X.M., Zhao, J., and David, J.H., Stability and L2-gain analysis for switched delay systems: A delay-dependent method, Automatica, Vol. 42(10)(2006), pp. 1769-1774.

[16] Xie, G, D.Z. Zheng D.Z.,On the controllability and reachability of a class of hybrid dynamical systems, Proc.19th Chinese Control Conference, Vol. 1(2000), pp. 114-117.

[17] Zhang, Y, Liu X , Zhu H, Zhong S., Stability analysis and control synthesis for a class of switched neutral systems, Applied Mathematics and Computation, Vol. 190(2007), pp. 1258-1266.

[18] Liu, J, Liu X.Z, Xie W., Delay-dependent robust control for uncertain switched systems with time-delay, Nonlinear Analysis: Hybrid Systems, Vol. 2(2008), pp. 81-95.

[19] Sun, X.M, Georgi M. Dimirovski, Zhao J, and Wang W, Exponential Stability for Switched Delay Systems Based on Average Dwell Time Technique and Lyapunov Function Method, American Control Conference Minneapolis, Minnesota, USA, June (2006),pp. 14-16.

[20] Gurvits, L , Stability of discrete linear inclusion, Linear Algebra and its Applications, Vol. 231(1995), pp. 47-85.

[21] Liu, X.Z., and Shen J.H, Stability Theory of Hybrid Dynamical Systems With Time Delay, IEEE Transactions on Automatic Control, Vol. 51(4)(2006), pp.620-625.

[22] Liberzon, D, Morse A.S, Basic problems in stability and design of switched systems, IEEE Contr. Syst. Mag, Vol. 19(1999), pp. 59-70.

[23] Zhai, G, Hu B, Yasuda K, and A. N. Michel, Stability and L2-gain analysis of discrete-time switched systems, Transactions of the Institute of Systems, Control and Information Engineers, Vol. 15(3)(2002), pp. 117-125.

[24] Solo, V, On the stability of slowly time-varying linear systems, Math. Contr. Signals Systems, Vol.7(4)(1994), pp. 331-350. 
[25] De Santis, E., Di Benedetto, M.D., Pola, G.Can linear stabilizability analysis be generalized to switching systems?.In: Proc. of Mathematical Theory of Networks and Systems (MTNS 2004), Leuven (Belgium), July 5-9 (2004).

[26] Lin, H., Antsaklis, P.J.Switching stabilizability for continuous-time uncertain switched linear systems. IEEE Trans. Aut. Control 52(2007), 633-646.

[27] Xiang, Z.R., Xiang, W.M.Stability analysis of switched systems under dynamical dwell time control approach. Int. J. Sys. Sci. 40(2009), 347355.

[28] Tarbouriech, S., Prieur, C., Gomes da Silva, J.M.:Stability analysis and stabilization of systems presenting nested saturations. IEEE Trans. Aut. Control 51, 1364-1371 (2006).

[29] Xie, G. M, Wang L., Controllability and stability of switched linearsystems. Systems and Control Letters, Vol.48(2)(2003), pp.135-155.

[30] Xie, G. M, Wang L, Stabilization of switched linear systems with time-delay in detection of switching signal, J. Math. Anal. Appl, Vol. 305(2005), pp.277-290.

[31] Halanay, A., Differential Equations: Stability, Oscillations, Time Lags. Academic, New York (1966).

[32] Boyd, S., L. El Ghaoui and V. Balakrishnan, Linear Matrix Inequalities in System and Control Theory, SIAM, Philadelphia, (1994).

[33] Yang, H., Jiang, B., Cocquempot V., and Zhang, H., Stabilization of switched nonlinear systems with all unstable modes: applications to multi-agent systems, IEEE Transactions on Automatic Control, Vol. 56(9)(2011), pp. 2230-2235.

[34] Yang, H., Jiang, B., On stability of non-linear and switched parabolic systems, IET Control Theory and Applications, 7(5)(2013), pp. 749-756.

[35] Yang, H., Jiang, B., and Zhang, H., Stabilization of non-minimum phase switched nonlinear systems with application to multi-agent systems, Sys-tems \& Control Letters, 61(10)(2012), pp. 1023-1031. 
[36] Zhang, L., Gao, H., Asynchronously switched control of switched linear systems with average dwell time, Automatica, Vol. 46, (5)(2010), pp. 953-958

[37] Mhaskar, P., El-Farra, N.H., Christofides, P.D., Robust predictive control of switched systems: satisfying uncertain schedules subject to state and control constraints, International Journal of Adaptive Control \& Signal Processing, Vol. 22(2)(2008), pp. 161-179

[38] Niu, B. and Zhao, J., Barrier Lyapunov functions for tracking control of constrained nonlinear switched systems, Systems \& Control Letters, Vol. 62(10)(2013), pp. 963-971.

[39] Niu, B. and Zhao, J., Robust $H_{\infty}$ control for a class of switched nonlinear systems with average dwell time, International Journal of Control, Vol. 86(6)(2013), pp. 1107-1117. 